\title{
Civilisations
}

Revue internationale d'anthropologie et de sciences

humaines

39 | 1991

Japon : les enjeux du futur

\section{Les formes de la pratique religieuse au Japon : persistance et changement}

\author{
Jean-Pierre Berthon
}

\section{(2) OpenEdition \\ Journals}

Édition électronique

URL : http://journals.openedition.org/civilisations/1642

DOI : 10.4000/civilisations. 1642

ISSN : 2032-0442

\section{Éditeur}

Institut de sociologie de l'Université Libre de Bruxelles

\section{Édition imprimée}

Date de publication : 30 octobre 1991

Pagination : 19-39

ISBN : 2-87263-044-9

ISSN : 0009-8140

Référence électronique

Jean-Pierre Berthon, "Les formes de la pratique religieuse au Japon : persistance et changement », Civilisations [En ligne], 39 | 1991, mis en ligne le 06 juillet 2009, consulté le 19 avril 2019. URL : http:// journals.openedition.org/civilisations/1642; DOI : 10.4000/civilisations.1642 


\title{
LES FORMES DE LA PRATIQUE RELIGIEUSE AU JAPON : PERSIST ANCE ET CHANGEMENT
}

\author{
Jean-Pierre BERTHON
}

\begin{abstract}
Le Japon connaît plusieurs traditions religieuses, populaires et savantes, qui, au cours des siècles, se sont mutuellement enrichies les unes les autres pour composer un syncrétisme religieux à double dominante, boudhique et shintoïque. Les particularités de ce syncrétisme sont plus à rechercher dans le cumul et l'addition d'éléments nouveaux en vue d'une tentative d'assimilation, que dans un véritable effort de synthèse débouchant sur une réinterprétation des sources premières'. Devant le caractère multiple du fait religieux et au sein d'une société longtemps déterminée dans sa culture religieuse par l'idée de panthéisme et par la prédominance de la pratique sur la doctrine, il paraît, à première vue, difficile de dresser un tableau synthétique des croyances et des pratiques religieuses qui puisse rendre compte d'une tendance générale unifiée. Nous essaierons, néanmoins, de mettre en lumière les transformations religieuses les plus marquantes, liées à de nouvelles conditions de vie - urbanisation, industrialisation, mouvements migratoires - sans pour autant négliger la nature des groupes ou des courants concernés.
\end{abstract}

\section{La société japonaise : une société sécularisée?}

S'interroger sur les changements religieux dans le Japon contemporain, c'est également se poser la question de l'importance des institutions et des comportements religieux à l'intérieur d'une société que certains décrivent comme dépourvue de sacré. Face à un Occident indifférencié, "désenchanté du monde", le Japon offrirait l'image stable d'une culture depuis longtemps laïcisée. Cette thèse semble être 
dominante à l'intérieur d'écrits - les Nihonjin-ron ou "Essais sur les Japonais" - qui visent à relever la spécificité et l'unicité de la culture japonaise dans ses particularismes les plus profonds, que ceux-ci soient sociaux ou culturels. C'est ainsi que la religion est convoquée comme illustration de ce point de vue. Dans un essai sur la mentalité japonaise ${ }^{2}$, Muramatsu insiste sur cette laïcité de la société japonaise qui remonte, selon l'auteur, au XVI siècle (incendie des monastères bouddhiques du mont Hiei par Oda Nobunaga). Elle trouverait sa forme présente, entre autres, dans le fait que les Japonais s'avèreraient incapables de répondre à la plus élémentaire des questions d'ordre religieux : "A quelle religion appartenezvous ?" Quant à. l'importance des groupements religieux, l'auteur ajoute: "Certes, les diverses formes du bouddhisme et quelques sectes shintoïstes de création plus récente conservent de fervents adeptes, mais le nombre de ceux-ci reste très limité"3. Intéressant est le fait que, parallèlement à ce phénomène de laïcisation, le même auteur cite, en illustration, certaines coutumes qui se seraient conservées, telle la croyance envers le paradis de la Terre pure (jödo) où l'homme irait renaître après la mort, ou bien la coutume de chausser le défunt d'une paire de sandales de paille (waraji) et de placer dans sa main un bâton de pèlerin.

Ce texte nous semble être un exemple de la difficulté d'appréhender le fait religieux à l'intérieur de la dichotomie modernité/tradition; le premier terme renvoyant à une société sécularisée, de moins en moins concernée par les institutions et les intérêts d'ordre religieux, en contraste avec la société traditionnelle où les croyances religieuses feraient encore partie intégrante de la conception du monde.

Que le processus de sécularisation ${ }^{4}$ de la société japonaise soit inévitable ou non, encore faut-il s'entendre sur son rythme de changement; en d'autres termes, et comme le suggère 
Winston (1980), le changement culturel - et en particulier religieux - n'est pas nécessairement synchrone avec les transformations d'ensemble de la société.

Une autre difficulté est celle de déterminer les indicateurs du processus de sécularisation (le nombre d'adhérents, le statut des organisations religieuses, la diminution des croyances envers des entités surnaturelles etc...). Est-ce la forme ou le contenu qui définit le changement d'un système religieux ? Pour revenir à la référence citée plus haut, quant à la preuve historique de l'ancienneté de la "sécularisation" de la société japonaise, on peut tout aussi bien se demander si le fait que le pouvoir politique n'ait jamais hésité à intervenir dans les affaires religieuses chaque fois qu'il se sentit menacé, ou bien que chaque famille influente ait divinisé ses ancêtres afin de légitimer son pourvoir temporel (Sonoda, 1983), ne serait pas l'expression - dans le cas japonais - d'une différenciation peu marquée entre société sacrée et société séculière ? Cette réflexion au niveau des institutions religieuses trouvant son prolongement naturel au niveau des idées et des croyances.

\section{Les structures de base de la religion japonaise}

Il n'y a jamais eu - comme nous le montre la présentation de l'histoire des religions au Japon - d'organisation unique du bouddhisme ou du shintoïsme, mais toujours plusieurs écoles de pensée qui donnèrent naissance à des courants divers. En dehors de toute présentation systématique de ces différentes écoles ou tendances, nous insisterons, en amont, sur l'unité générale de la religion japonaise, avant d'aborder la diversité des cultes et des croyances tels qu'ils se présentent dans le Japon d'aujourd'hui.

La religion japonaise s'exprime à travers des façons de vivre et de mourir que nous allons essayer de mettre en 
évidence à travers la diversité de l'expérience religieuse. Cette vision du monde particulière, diversement notée par nombre d'auteurs, s'ancre autour de cinq points caractéristiques :

a) observances tournées vers le monde présent;

b) proximité et complémentarité des notions de sacré et de profane;

c) centralité du rite et primauté de l'expérience directe;

d) pénétration de la religion dans la vie quotidiennne;

e) fonction religieuse de la famille.

2.1. Les premiers écrits qui nous renseignent sur les croyances japonaises à l'époque ancienne - les Annales du début du VIII (Kojiki, 712) et Nihon Shoki, 720) - ne font guère mention de préoccupations tournées vers l'autre monde. Le séjour des morts est un lieu de pourriture et de décomposition. Des rites de purification - par l'eau ou par le feu - permettent de se tenir à distance de ce monde infernal. Ce qui est central dans les préoccupations quotidiennes, ce sont les notions de pureté et d'évitement.

Par ailleurs, si le bouddhisme contient des descriptions édifiantes de l'au-delà et peut apparaître - sous sa forme amidiste (sectes de la Terre Pure) - comme une religion de salut, c'est surtout à travers les rites d'exorcisme qu'il se répand dans le peuple. Les divinités conçues comme dispensatrices de bienfaits, comme protectrices des biens et des âmes, donnent aux rites leurs colorations particulières. Que la réalité ultime soit à rechercher dans cette vie, et non dans la vie future, n'est pas sans relation avec le $2^{\mathrm{e}}$ point, à savoir, la proximité du sacré.

2.2. Dans l'univers religieux japonais n'existe pas de ligne de démarcation bien nette entre sacré et profane. La relation harmonieuse triangulaire, homme-nature-divinités, participe 
d'une représentation du monde à l'intérieur de laquelle existent une interaction permanente entre les humains et les nonhumains, entre des forces négatives et des forces positives, et une proximité de nature entre les kami (divinités autochtones) et les ancêtres. Les ikigami (mot à mot "divinité vivante") terme qui désigne plus spécialement certains fondateurs de nouvelles religions -, de même que certains patriarches du bouddhisme (secte Ji), dénommés ikibotoke ("bouddha vivant"), en sont deux exemples, comme en sont également un autre exemple, les personnages illustres divinisés. Citons le plus célèbre d'entre eux, Sugawara Michizane - lettré de l'époque Heian - divinisé sous les traits de la divinité Tenjin (divinité de l'abondance) et vénéré au sanctuaire shintoïque, Kitano Tenman-gû, de Kyôto.

Du point de vue des croyances, la conception bouddhique selon laquelle la Nature du Bouddha est contenue en toute chose (isshaishujö shitsuubusshô), conférait au monde séculier (seken ) une essence sacrée. Cet "enchevêtrement" (kattô) du sacré et du profane fut mis en évidence par Hori (1975) dans un essai sur l'applicabilité du terme sezokuka - traduction japonaise de "sécularisation" - à l'histoire des religions au Japon.

2.3. Centralité du rite et primauté de l'expérience directe. Plus qu'à travers un ensemble de croyances et de corps de doctrines, la religion japonaise est avant tout vécue par et dans l'exercice rituel. Rites collectifs ou individuels, exercices ésotériques ou ascétiques en vue d'obtenir des pouvoirs magiques, la pratique rituelle est la "voie" essentielle de l'homo religiosus japonais. L'expérience immédiate, école subitiste dans le bouddhisme zen (secte rinzai) ou encore révélations divines par l'intermédiaire de personnages à tendances chamaniques, est une autre particularité de la pratique religieuse japonaise. 
2.4. Pénétration de la religion dans la vie quotidienne. Les offrandes quotidiennes aux multiples divinités vénérées, les nombreuses visites aux temples et aux sanctuaires - visites accomplies en considération de rites saisonniers ou des différentes étapes de la vie humaine - sont la marque d'une religion vécue dans sa dimension quotidienne. Il n'y a pas jusqu'à l'espace rituel qui ne reflète cette tendance. En effet, à l'époque ancienne, le lieu de déroulement du rituel était un lieu quotidien. Il n'existait pas de lieux sacrés permanents. Le rite accompli, l'autel était détruit et l'endroit rendu à la vie profane. L'émergence d'un espace sacré à l'intérieur de l'espace public se manifeste encore aujourd'hui à travers le torii (portail du sanctuaire shintö) ou la corde cérémonielle (shimenawa) qui délimite, pour un temps, un espace sacré. Les zones liminaires, sans délimitation bien tranchée, forment des lieux imprégnés de sacralité.

2.5. Fonction religieuse de la famille. La famille japonaise traditionnelle (ie ) est à la fois un groupe de parenté, un groupe économique et un groupe religieux. La cohésion de la maison principale (honke) et de ses maisons détachées (bunke) était réalisée à travers des activités rituelles agraires et des rites dédiés aux ancêtres de la maison fondatrice. Une répartition fonctionnelle de ces rites s'établissait autour du shintoïsme (cultes agraires) et du bouddhisme (cultes rendus aux esprits défunts). Ce dernier type de rite contribuait à la pérennité de la maison mère, souci majeur de tous ses membres. Cette fonction intégrative du ie semble avoir joué au Japon un rôle semblable à celui de l'Eglise dans les sociétés européennes, d'où l'importance d'accorder à cette institution et au culte des ancêtres qui en est l'une des composantes principales la considération qu'ils méritent ${ }^{5}$. 


\section{La mesure de l'intensité religieuse}

Dans le Japon urbain et post-industriel d'aujourd'hui, les statistiques concernant la pratique et la foi religieuses révèlent à la fois, un foisonnement des rites et des fêtes, une recrudescence des pratiques dites magiques ("le retour du religieux" ?, Komot et alii, 1985), et une diminution du poids des grandes institutions religieuses shintoïques et bouddhiques, si tant est qu'elles aient jamais eu une réelle influence sociale.

Sous quelles formes, suivant quels canaux, les comportements et sentiments religieux s'expriment-ils?

Quelques chiffres nous aideront à cerner de plus près la notion si fluide de religiosité à travers les 88.965.060 bouddhistes et les 112.106.715 shintoïstes que compterait le Japon $^{6}$ (un rapport de 1,8 avec l'estimation de la population japonaise).

C'est ainsi que :

- $65 \%$ des personnes interrogées affirment être dépourvu de foi religieuse (shinkô ) mais :

- $53 \%$ d'entre elles rendent un culte quotidien ou accasionnel, devant l'autel domestique shintô (kamidana) et $57 \%$ devant l'autel domestique bouddhique (butsudan)

- $81 \%$ se rendent à un sanctuaire ou à un temple lors des premiers jours de l'année nouvelle (hatsumôde); $89 \%$ visitent les tombes de leurs ancêtres au moment de la fête des morts (o-bon) et lors des fêtes bouddhiques d'équinoxe (higan) de printemps et d'automne

- $77 \%$ se procurent des charmes et des amulettes auprès des centres religieux. 
A quels référents renvoie cette sensibilité religieuse ? Ils sont essentiellement au nombre de cinq, comme l'enquête le souligne:

a) le culte des ancêtres (59\% y sont profondément attachés, $71 \%$ possèdent un autel domestique bouddhique);

b) le sentiment de fragilité de l'existence humaine. Cette faiblesse mentionnée par $85 \%$ des personnes interrogées, est ressentie de manière égale par l'ensemble des classes d'âge considérées;

c) la soumission à son sort. Cette pensée fataliste, quant à la possibilité de surmonter un obstacle par ses propres forces, est exprimée par $86 \%$ des personnes;

d) la rétribution selon la loi de la causalité karmique (inga ôhô ). $87 \%$ de personnes pensent que des actes mauvais entraînent des effets néfastes et des actes bons, des récompenses similaires. L'origine bouddhique de ce terme ne doit pas faire oublier que cette tendance souligne la permanence très profonde d'un sentiment religieux partagé par la quasi totalité de la population, même si le pourcentage est plus élevé pour les personnes âgées, et à l'intérieur de ces dernières, pour les femmes.

e) dernier point commun, l'attente de "bénéfices immédiats" (genze riyaku ). $60 \%$ s'adressent aux divinités bouddhiques et shintoïques en cas de besoins non satisfaits; $54 \%$ pensent que leurs voeux seront exaucés.

\section{Les formes de la pratique religieuse}

\subsection{Les cultes domestiques}

L'espace domestique reste le lieu par excellence des observances quotidiennes. Autrefois plus nombreux et plus prégnants, les rites domestiques s'adressaient à diverses divinités (okunaishin) ayant chacune leur lieu de résidence propre : Ebisu et daikoku - divinités des rizières - dans la chambre des époux, Kôjin, Okamasama - divinités protectrices 
contre les incendies - dans la cuisine, Benjôgami, dans les toilettes. Chacune de ces pièces comprenait, à l'origine, un autel domestique.

Actuellement deux autels, l'un d'origine shintô (le kamidana ), l'autre d'origine bouddhique (le butsudan), sont l'expression matérielle de la religiosité quotidienne.

Selon une enquête nationale de $1981^{1}, 61 \%$ des personnes interrogées possèdaient un butsudan et $60 \%$ un kamidana. L'existence du kamidana est récente et doit être probablement mise en rapport avec, d'une part, la coutume des pèlerinages à Ise et la distribution de talismans, et d'autre part, les mesures de politique religieuse mises en place après la Restauration Meiji (1868) et visant à faire du shintô une religion nationale. En effet, selon les croyances religieuses à l'encontre des kami, ces derniers ne résident pas en permanence dans le même emplacement. A l'exception des autels temporaires, établis au moment des grandes fêtes calendaires (passage de l'an, fête des morts) et qui donnent lieu à des rites spécifiques, le culte devant l'autel domestique shintô consiste principalement et simplement, à remplacer l'offrande quotidienne et à réciter une courte prière.

L'origine du butsudan est plus ancienne. Elle est le résultat d'une double transformation : aux petits temples privés des nobles de l'époque de Heian (794-1185) à l'intérieur desquels était honorée une image bouddhique - souvent celle du fondateur de la secte à laquelle appartenait la famille -, se substitua, peu à peu, un autel devenu lieu de culte des esprits défunts; d'autre part, c'est à la faveur de l'enregistrement obligatoire de la population à un temple bouddhique (au XVII ${ }^{e}$ ) que le butsudan pénétra à l'intérieur des maisons. Dans le lent processus que constitue le phénomène de l'ancestralisation, le culte quotidien rendu devant l'autel bouddhique est un rite 
essentiel. Il marque la proximité et l'interaction des vivants et des morts. Le butsudan contient, entre autres, les tablettes mortuaires (ihai) des membres de la "maison". Tout comme le kamidana, il est l'objet de soins quotidiens. De plus, au moment des temps forts de l'année bouddhique (fête des morts, équinoxes de printemps et d'automne, jour anniversaire et jours commémoratifs), il est le centre de rites élaborés qui requièrent la présence du "moine de famille"8.

En dehors de ces cultes domestiques, d'autres cultes sont rendus à l'intérieur de l'espace villageois. Nous citerons les cultes aux divinités de la résidence et du site d'habitation (terme générique de yashiki-gami ), les cultes aux divinités protectrices du village ou à celles qui résident aux limites de ce dernier (Kannon, Jizô, dôsojin), enfin les divinités tutélaires honorées au sanctuaire villageois.

\subsection{Les "fêtes" (matsuri)}

Les matsuri sont, stricto sensu, des cérémonies religieuses qui consistent à honorer une divinité shintô, généralement la divinité principale du sanctuaire villageois. Si la fonction religieuse des matsuri a perdu de sa vigueur (on a vu récemment se mettre en place des fêtes sans divinité ou aux "divinités anonymes), ces manifestations n'en continuent pas moins de jouer un rôle essentiel : intégrer l'individu au groupe et renforcer le sentiment de solidarité communautaire, essentiel pour une ancienne société rizicole où l'entraide était indispensable. C'est encore aujourd'hui la fonction principale des innombrables fêtes de quartier en milieu urbain.

Le déroulement de la "fête" est semblable à celui de tout culte rendu à l'encontre d'une divinité et ne diffère pas fondamentalement des rites domestiques ou des visites aux temples et aux sanctuaires. Il se décompose comme suit : 
- les préparatifs avec, pour les officiants (desservants du sanctuaire, mais aussi, parfois, les villageois eux-mêmes), le respect de divers interdits qui se sont allégés au fil du temps. La plus grande pureté est requise avant l'accomplissement de la "fête" : purification par l'eau (misogi) ou par un rite d'exorcisme (o-harai);

- l'accueil de la divinité. Pour que la divinité consente à descendre sur le site choisi divers supports sont utilisés. Originellement il s'agissait d'objets naturels (pierres, plantes, monticules, voire montagnes) ou même d'animaux souvent considérés comme les messagers de la divinité ou manifestation temporaire de celle-ci. Les kannushi (desservants) utilisent l'objet appelé gohei (bâton muni en son extrémité de bandes de papier blanc). Les êtres humains enfin servent de relais entre les villageois et la divinité. Il s'agissait, à l'origine, de médiums (milo) attachés au sanctuaire, capables d'entrer en transe, de convoquer les kami et de transmettre leurs messages;

- la présentation d'offrandes et la récitation de prières rituelles (norito ). Les offrandes présentées seront partagées lors du repas pris en commun (naorai) qui clôt la "fête". La récitation des norito (textes très anciens dont l'origine incertaine est déjà attesté au VIII ${ }^{e}$ siècle) avait pour but, en comblant d'éloges la divinité, de s'assurer de sa protection et de demander l'abondance "de ces produits de la mer et de la montagne";

- l'acquisition de la force divine. Le repas de clôture permet, de plus, aux habitants de recevoir une part de l'énergie vitale de la divinité;

- le renvoi de la divinité. Il répond à l'accueil de celle-ci au début du matsuri. On raccompagne la divinité dans sa demeure provisoire, le sanctuaire shintô;

- la fête est également l'occasion de réjouir la divinité honorée, par des spectacles de danse (kagura), des compétitions sportives (luttes de sumô, luttes à la corde, tir à l'arc), des 
représentations théâtrales (spectacle de nô, musique de Cour, danses et chants paysans).

Qu'en est-il aujourd'hui ? Si les adresses aux dieux pour se prémunir contre les maladies ou les calamités naturelles ont perdu de leur raison d'être, le sentiment de participer à la consolidation de liens, distendus par le mode de vie citadin, reste toujours très fort.

Les matsuri attirent souvent des personnes sans lien avec le lieu de déroulement de la cérémonie. Les sanctuaires shintô situés en milieu urbain exercent des fonctions multiples correspondant à divers "types de paroissiens" (permanents, occasionnels, nouveaux). A la place des sanctuaires tutélaires dont la population religieuse constituait les enfants (ujika), les fêtes des sanctuaires qui continuent à maintenir une activité religieuse importante sont ceux qui parviennent à draîner une population extérieure, grâce aux vertus imminentes de leurs divinités, ou bien au prestige du sanctuaire lui-même. Les grandes fêtes annuelles qui renforçaient la cohésion sociale ont été maintenues pour l'essentiel, mais ne bénéficient plus guère du soutien actif de groupes de jeunes.

A la ville, d'anciennes fêtes sont remises au goût du jour, voire même créées de toutes pièces comme la célèbre Kôbe matsuri, en $1971^{8}$. Il arrive que les dates de célébration soient modifiées afin de coïncider avec des jours fériés.

Absents de certaines fêtes profanes, les kami se réfugient parfois à l'intérieur des entreprises japonaises, soit sous la forme de sanctuaires d'entreprises (kigyô jinja ) florissants, soit par l'indispensable contribution financière qu'apportent les sociétés de commerce aux sanctuaires environnants, par exemple, au quartier de Ginza, à Tôkyô (Ishii, 1986b). Les rites religieux, au sens large du terme, sont également la marque de 
nombre d'entreprises qui voient en eux la consolidation d'une éthique spécifique (Waki, 1984).

\subsection{Les pèlerinages et autres voies d'ascèse}

Le pèlerinage semble avoir été, à l'origine, un phénomène essentiellement bouddhique. Le terme qui désigne d'ailleurs en japonais le pèlerinage - junrei (circuit de dévotions) - est un terme bouddhique comme le sont aussi ses autres appelations: henro (cheminement), kaikoku (tour des provinces).

En effet, les croyances religieuses pré-bouddhiques voulaient que ce soient les divinités tutélaires du village qui veillent sur ses habitants; il n'était donc pas nécessaire d'aller chercher protection dans un autre lieu. A cela s'ajoute le fait que la pénétration des montagnes (endroits privilégiés de pèlerinage, mais aussi lieux de séjour des morts) étaient, excepté pour eux qui y travaillaient, des lieux inhospitaliers, interdits d'accès et taboués.

Le syncrétisme shintô-bouddhique marqua l'avènement des premiers grands pèlerinages. Ainsi, le pèlerinage le plus célèbre du pays, celui aux trois montagnes de Kumano (Kumano sanzan), tire son essence de la dévotion aux kami des montagnes, respectivement assimilés aux trois divinités bouddhiques qui leurs correspondent, à savoir: Amida, Kannon, Yakushi. Le but du pèlerinage est double : s'assurer, par une longue et difficile pratique ascétique, des bénéfices dans le monde présent (shintô) et une renaissance avantageuse dans l'un des paradis bouddhiques. Ce syncrétisme qui montre à l'évidence la forte influence du bouddhisme dans l'univers religieux japonais, ne doit pas faire oublier que certains lieux sacrés shintô resteront très longtemps à l'abri de cette influence. Le grand sanctuaire d'Ise échappa ainsi, à l'époque ancienne, à 
la pénétration du bouddhisme (accès interdit à tout moine bouddhique, tabou de toute formule bouddhique prononcée).

Aujourd'hui, les pèlerinages majeurs sont ceux de Shikoku (88 stations visitées, dit-on, en compagnie du saint bouddhique qui aurait le premier prié en ces sites - Kôbô Daishi, fondateur de la secte Shingon, du bouddhisme ésotérique -) et des "Provinces occidentales" - Saikoku - où chacun des trente-trois monastères visités renferme une image de la divinité de la miséricorde, Kannon bosatsu. Il est à noter que ces circuits de l'ouest du Japon (région du Kinki et de Shikoku) se doublent de leurs pendants dans le Japon de l'est (région de Tôkyô). Le pèlerinage shinto le plus important est, sans conteste, celui à Ise (Ise-mairi). Il permettait à une population politiquement maintenue à l'intérieur d'un système rigide de contrôles et de réglementations, de quitter son univers villageois pour aller honorer les ancêtres de la Maison impériale.

Outre leur caractère sacré, les pèlerinages ont toujours été, au Japon, prétexte à promenade et à réjouissance (l'étude d'un carnet de voyage à Ise, au XVII siècle, a révélé que sur la centaine de jours que dura le pèlerinage, seuls trois furent effectivement passés à Ise, les autres s'étant écoulé dans les stations thermales et autres lieux touristiques).

Les temples et sanctuaires à la mode (la presse féminine n'y est pas étrangère), les innombrables circuits de dimension plus réduite, voire même le "tour des 7 divinités du bonheur" pendant les premiers jours de l'an, attirent une foule considérable, souvent jeune. A ces pèlerinages doivent être ajoutés, pour la même période, la coutume du hatsu-môde (ou première visite à un temple ou à un sanctuaire). Selon l'enquête de $1984,81 \%$ de la population japonaise se rendrait dans l'un de ces lieux saints; ce qui, pour les plus importants d'entre eux, correspond à plusieurs millions de visiteurs. 
Les pratiques ascétiques qui se sont maintenues dans le Japon contemporain (prescriptions rituelles, purification par l'eau ou le sel, séjour en montagne à travers l'appartenance à une confrérie) sont la trace de "voies" anciennes également partagées à l'intérieur du bouddhisme et du shintoïsme, mais aussi et surtout, dans la "petite tradition" (moines et ascètes itinérants, pratiquants du shugendô - "voie" ( $d \hat{o}$ ) en vue d'acquérir par la pratique ascétique $(s h u)$ une efficacité miraculeuse (gen )").

\subsection{L'acquisition d'amulettes et la divination}

Selon l'enquête précédemment citée, $77 \%$ des personnes interrogées déclarent se procurer, souvent (43\%), ou occasionnellement ( $34 \%)$, des amulettes et des talismans auprès des centres religieux.

Une répartition schématique de ces objets permet de mentionner ceux qui visent à se protéger d'influences néfastes pour l'individu ou le groupe : o-mamori (amulette protectrice), et ceux qui donnent un pouvoir et/ou un contrôle en vue d'acquérir un bénéfice : $o$-fuda (talisman). Les o-mamori étaient, à l'origine, des objets naturels tels que du sable ou une pierre d'un sanctuaire renommé; ces objets étaient enfermés dans le sac du voyageur et visaient à assurer sa protection jusqu'à son retour.

Certains o-mamori consistent en tablettes protectrices émises par des temples ou des sanctuaires. Elles protègent des calamités naturelles, des maladies (variole, rougeole) et à l'encontre des enfants, tout spécialement, assurent leur bonne croissance.

Les $o$-fuda sont fabriquées par les temples et les sanctuaires; ils comportent toujours une inscription et une 
image imprimée sur une tablette ou une feuille de papier. Il s'agit généralement de la divinité vénérée dans le lieu d'acquisition. L'o-fuda spécifie clairement le but pour lequel il a été émis (protection contre les incendies et les vols); il contient en général une représentation symbolique de la puissance divine dont il est investi.

Placés selon leur provenance sur l'autel domestique shintô ou bouddhique, les $o-f u d a$ sont objets de vénération et de prières. Selon le principe de la "division des divinités" (bunrei, bunshin ), ils sont considérés comme des doubles de ces dernières.

A l'origine, les $o$-fuda semblent avoir assuré une protection collective (bâtiments, communauté villageoise, rizières) contre des divinités pourvoyeuses de maladie, contre les voleurs et toutes sortes d'influences néfastes. Ils appartiennent à la catégorie générale de "chasse-démons" (mayoke). Ces talismans étaient apposés en certains lieux précis (piliers, portes, limites villageoises) et attestaient de cette protection divine. Il arrive que les pèlerins, à leur tour, fassent don - à titre personnel ou au nom de la communauté villageoise - d'un ofuda au temple ou au sanctuaire visité; il est dans ce cas généralement collé sur la porte d'entrée ou sur le pilier de la salle des prières.

O-mamori et $o$-fuda portent la marque de la tradition syncrétique de l'univers religieux japonais, qu'il s'agisse des inscriptions incantatoires des premiers, ou bien des noms des divinités, de la formule, ou du titre du sûtra porté sur les seconds. L'influence du taoïsme religieux semble forte dans le cas des o-mamori, où certaines inscriptions reproduisent des formules de décrets officiels de la dynastie chinoise des Han. Célèbres entre toutes sont les amulettes des temples et sanctuaires de la province du Kinki, dénommées goô hôin 
(littéralement "sceau précieux de la divinité bovine"). Distribuées au seuil du premier ou du deuxième mois de l'année, elles assurent protection pour la durée de l'année nouvelle.

Les amulettes renferment trois sortes de pouvoir : la protection (shugo), la guérison (chibyô) et l'acquisition de "bénéfices immédiats dans le monde présent" (genze riyaku). Leur efficacité s'amenuise à mesure que l'année s'avance, par suite des impuretés accumulées. C'est ainsi que périodiquement - le plus souvent au début du sixième mois, à l'année nouvelle ou bien le jour de la fête du sanctuaire ou du temple - les amulettes sont jetées et brûlées, ou bien renvoyées sur des embarcations, au fil de l'eau. Les visites des premiers jours de l'an (hatsumôde), ou bien le pèlerinage à un lieu de culte célèbre sont l'occasion de renouveler les amulettes.

La pratique de la divination, qui occupait une place prépondérante dans la société paysanne (interprétation de phénomènes météorologiques en rapport avec le travail de la terre - signe auspicieux de la neige du début de l'an -) ainsi que dans la société aristocratique de Heian (794-1185) à travers l'Office de divination, est encore aujourd'hui une pratique très vivante. Les anciens devins professionnels de l'époque d'Edo (1603-1868) - les ekisha - sont, bien qu'en nombre restreint, toujours appréciés. Ils officient principalement dans l'enceinte des temples et des sanctuaires. Si la pratique de l'uranai (divination à l'aide de divers supports tels que pierres, pâte de riz, haricots) est de moins en moins courante ( $23 \%$ de personnes interrogées) celle du $o$-mikuji (consultation des horoscopes) dans les sanctuaires shintô, au moment des examens ou du mariage, est très populaire (57\% des personnes interrogées s'y adonnent). 


\section{NOTES}

1. Pour une réflexion globale sur la notion de syncrétisme religieux, à travers des exemples empruntés à des aires géographiques diverses, voir Augé 1988; pour le cas japonais, voir Rotermund, 1988.

2. Muramatsu T., 1974.

3. Idem, p. 5-6.

4. Sur le phénomène de la sécularisation, voir Shiner, 1967 et Martin, 1978. Pour une réflexion et une application de ce concept dans le contexte de la société japonaise, voir Ikado, 1972; Swyngedouw, 1979; Tamaru, 1987; Winston, 1980. Pour la spécificité du processus selon la tradition religieuse, voir Ishii, 1986a (bouddhisme) et Ueda, 1979 (shintô).

5. Pour une introduction à la notion de ie et à l'importance de sa fonction dans la société japonaise, voir Beillevaire, 1986; Morioka, 1981 et 1986, offre une image contrastée d'une structure et d'un culte qui se modifient sous l'effet conjugué de l'urbanistion et de l'industrialisation.

6. Nous nous appuyons sur les résultats d'une enquête systématique effectuée tous les cinq ans, par la N.H.K. (N.H.K. Seron chôsa-bu, 1984). Le nombre de personnes interrogées était, pour l'enquête de 1984, de 3.600. Les chiffres concernant la population religieuse sont tirés de l'Annuaire des religions publié par la section des Affaires religieuses de l'Agence des Affaires culturelles (Bunka-chô, 1986).

7. N.H.K. Seron chôsa-bu, 1981.

8. Pour une répartition spatiale des deux autels domestiques prenant en compte la dichotomie ville/campagne, voir Nakamaki, 1983.

9. Voir l'étude consacrée à cette fête par un groupe de sociologues (Inoue et alii, 1979). 


\section{REFERENCES}

AUGE, M., 1988, "Les syncrétismes "in Encyclopaedia Universalis (ed), Le grand atlas des religions, Paris, p. 130-131.

BEILLEVAIRE, P., 1986, "Le Japon, une société de la maison" in, Histoire de la famille, vol. 1, Paris, A. Collin, p. 479-517.

BUNKACHO (ed), 1986, Shukyô nenkan, Tôkyô, Gyôsei.

HORI, I., 1975, Sei to zoku no kattô , Tôkyô, heibonsha.

INOUE, N. et alii, 1979, "A Festival with Anonymous Kami : The Kobe Matsuri", Japanese Journal of religious Studies, vol. 6, n 1-2, p. 163-185.

ISHII, K., 1986a "Sezoku shakai ni okeru bukkyô no kanôsei", Risô , n 76. pp. 168-176. 1986b, "The Secularization of religion in the City", Japanese Journal of religious Studies.

KOMOTO, M. et alii, 1985, "Shûkyô kaiki ?" Shinri to sôzô, n² 24, Tôkyô, p. 2-41.

MARTIN, D.A., 1978, A General Theory of Secularization, Oxford, Basic Blackwell.

MORIOKA, K, 1986, "Ancestor Worship in Contemporary Japan : Continuity and Change", Senri Ethnological Studies, $\mathrm{n}^{\circ} 11$, Senri, Osaka, p. 201-213 (DEVOS, G, SOFUE T (eds) Religion and Family in East Asia).

MORIOKA, K. (ed), 1986, Kingendai ni okeru 'ie' no henshitsu to shûkyô , Tôkyô, Shinchi Shobô.

MURAMATSU, T., 1974, "Outline of the Japanese Way of Thinking", Information Bulletin, 1974, Ministère des Affaires Etrangères, Tokyo (Extraits traduits par la Documentation Française, Japon : Tradition et développement, P.P.S. sécire Extrême-Orient, $\mathrm{n}^{\circ}$ 278, p. 4-8). 
NAKAMAKI, H., 1983, "The 'Separate' Coexistence of Kami and Hotoke : A Look at Yorishiro", Japanese Journal of Religious Studies, vol. 10, $\mathrm{n}^{\circ} 1$, p. 65-86.

NHK SERON CHOSA-BU (ed), 1981 et 1984, Nihonjin no shûkyô ishiki, Tôkyô, Nihon hôsô shuppan kyôkai.

ROTERMUND, H.O., 1988, "Le syncrétisme au Japon", in Encyclopaedia Universalis (ed), Le grand atlas des religions, Paris, 136-137.

SHINER, L., 1967, "The Concept of Secularization in Empirical research", Journal for the Scientific Study of Religion, vol. 6, p. 207-220.

SONODA, M., 1983, "Secularity and Profanation in Japanese Religion", in Institute for Japanese Culture and Classics (ed) Cultural Identity and Modernization in Asian Countries, Tokyo, Kokugakuin University, p. 75-85.

SWYNGEDOUW, J., 1979, "Reflections on the Secularization Thesis in the Sociology of Religion in Japan", in Proceedings of the 1978 Tokyo meeting of the conférence internationale de sociologie religieuse, Tokyo, p. 65-88.

TAMARU, N., 1987, "The Concept of Secularization and its Relevance in Japanese Society", The Journal of Oriental Studies, vol. $26, \mathrm{n}^{\circ} 1, \mathrm{pp}$. 51-61 (Feature : Beyond the Dichotomy of Secularity and Religion).

UEDA, K., 1979, "Contemporary Social Change and Shinto Tradition", in Proceedings of the 1978 Tokyo meeting of the conférence internationale de sociologie religieuse, Tokyo, p. 303-327.

WAKI, H., 1984, "Kamigami no dôin", Asahi shinbun, 28 janvier (édition du matin).

WINSTON, D., 1980, "The Secularization of Japanese Religion : Measuring the Myth and the Reality", in F. REYNOLDS \& Th. M. LUDWIG (eds) Transitions and Transformations in the History of Religions, Leiden, p. 259-285. 


\section{SUMMARY}

Japan has a number of religious tradition, popular and learned, which have enriched each other over centuries. This forms a religious syncretism with a double axis, buddhist and shintoist. The particularity of this syncretism is that it results more from an accumulation and addition of new elements in view of assimilation that from an attempt of synthesis leading to a new interpretation of the primary sources. 